\title{
Efeitos da melatonina como tratamento complementar de úlceras gastroduodenais:
}

\section{Uma revisão integrativa}

\author{
Effects of melatonin as a complementary treatment for gastroduodenal ulcers: An integrative \\ review \\ Efectos de la melatonina como tratamiento complementario de las úlceras gastroduodenales: Una \\ revisión integradora
}

Recebido: 14/05/2021 | Revisado: 19/05/2021 | Aceito: 22/05/2021 | Publicado: 08/06/2021

\author{
Virgínia Raquel dos Santos Lucena \\ ORCID: https://orcid.org/0000-0003-3526-245X \\ Universidade Federal do Rio Grande do Norte, Brasil \\ E-mail: virginia.lucena.017@ufrn.edu.br \\ Rícia Medeiros Palmeira de Araujo \\ ORCID: https://orcid.org/0000-0002-4158-0748 \\ Universidade Federal do Rio Grande do Norte, Brasil \\ E-mail: ricia.palmeira.065@ufrn.edu.br \\ Deborah Laís Nóbrega de Medeiros \\ ORCID: https://orcid.org/0000-0003-4344-1984 \\ Universidade Federal do Rio Grande do Norte, Brasil \\ Email: deborah.nobrega.132@ufrn.edu.br \\ Maria Fernanda Almeida Silva \\ ORCID: https://orcid.org/0000-0002-8660-079X \\ Universidade Federal do Rio Grande do Norte, Brasil \\ Email: maria.silva.014@ufrn.edu.br \\ Sebastião Duque Neto \\ ORCID: https://orcid.org/0000-0002-5569-7568 \\ Universidade Federal do Rio Grande do Norte, Brasil \\ Email: sebastiaopdn@yahoo.com.br
}

\begin{abstract}
Resumo
Introdução: É conhecida a influência da ritmicidade biológica na regulação de diversos sistemas, incluindo o gastrointestinal. Nesse contexto, vários estudos recentes relacionam a liberação de melatonina, como uma substância importante na cicatrização de lesões ulcerativas no trato gastrointestinal. Objetivo: Investigar estudos e evidências acerca do papel da melatonina como tratamento complementar de úlceras gastroduodenais e sua relação com a cronobiologia. Metodologia: Trata-se de um estudo de revisão integrativa, realizada por meio de busca ativa nas bases de dados Scientific Eletronic Library Online (SciELO), PubMed, Science Direct e Literatura Latino Americana e do Caribe em Ciências da Saúde (LILACS) em agosto de 2020. Resultados: Em vários dos estudos analisados, a administração de melatonina mostrou-se eficaz e segura para acelerar a taxa de cicatrização das úlceras gastroduodenais de distintas etiologias por suas atividades antioxidante e anti-inflamatórias. Conclusão: Embora existam inúmeras evidências, a literatura é a ainda escassa quanto à realização do tratamento ou pré-tratamento das úlceras com melatonina, seja isoladamente ou em conjunto com outras drogas.
\end{abstract}

Palavras-chave: Cronobiologia; Melatonina; Úlceras gastroduodenais.

\begin{abstract}
Introduction: The influence of biological rhythmicity on the regulation of several systems, including the gastrointestinal system, is well known. In this context, several recent studies relate the release of melatonin, as an important substance in the healing of ulcerative lesions in the gastrointestinal tract. Objective: To investigate studies and evidence about the role of melatonin as a complementary treatment for gastroduodenal ulcers and its relationship with chronobiology. Methodology: This is an integrative review study, carried out through an active search in the Scientific Eletronic Library Online (SciELO), PubMed, Science Direct and Latin American and Caribbean Literature in Health Sciences (LILACS) databases in August 2020. Results: In several of the studies analyzed, the administration of melatonin proved to be effective and safe to accelerate the rate of healing of gastroduodenal ulcers of different etiologies due to its antioxidant and anti-inflammatory activities. Conclusion: Although there is innumerable evidence, the literature is still scarce regarding the treatment or pre-treatment of ulcers with melatonin, either alone or in conjunction with other drugs.
\end{abstract}

Keywords: Chronobiology; Melatonin; Gastroduodenal ulcers. 


\section{Resumen}

Introducción: Es bien conocida la influencia de la ritmicidad biológica en la regulación de varios sistemas, incluido el gastrointestinal. En este contexto, varios estudios recientes relacionan la liberación de melatonina, como una sustancia importante en la cicatrización de lesiones ulcerativas en el tracto gastrointestinal. Objetivo: investigar estudios y evidencias sobre el papel de la melatonina como tratamiento complementario de las úlceras gastroduodenales y su relación con la cronobiología. Metodología: Se trata de un estudio de revisión integradora, realizado a través de una búsqueda activa en las bases de datos Scientific Eletronic Library Online (SciELO), PubMed, Science Direct y Latin American and Caribbean Literature in Health Sciences (LILACS) en agosto de 2020. Resultados: En varios De los estudios analizados, la administración de melatonina demostró ser eficaz y segura para acelerar la tasa de curación de úlceras gastroduodenales de diferentes etiologías debido a sus actividades antioxidantes y antiinflamatorias. Conclusión: Aunque existe una innumerable evidencia, la literatura aún es escasa sobre el tratamiento o pretratamiento de las úlceras con melatonina, ya sea sola o en conjunto con otros fármacos.

Palabras clave: Cronobiología; Melatonina; Úlceras gastroduodenales.

\section{Introdução}

O primeiro registro de caso de úlcera gastroduodenal remonta a 460 a.C., sendo o diagnóstico atribuído a Hipócrates. No século XVIII, conhecido como "século das luzes", o amplo avanço na medicina legal permitiu o registro de úlceras perfuradas como causas raras de morte. Tal situação tornou-se mais comum nos séculos XIX e XX, e muitos pesquisadores atribuíram equivocadamente, então, a fatores ambientais da época como aumento do estresse, urbanização intensa, guerras mundiais, crise econômica e intensa industrialização (Susser \& Stein, 1962). O fato das úlceras gástricas e duodenais serem cada vez mais descritas no final século XIX, atingindo um pico na década de 1950, deve-se segundo Majumdar e Bebb (2011) o Helicobacter pylori tenha sido onipresente na população humana por milhares de anos.

Posteriormente, a patogênese da úlcera foi melhor elucidada em 1982, quando os cientistas Warren e Marshall isolaram a bactéria Helicobacter pylori da mucosa gástrica de pacientes com úlceras (Majumdar, Bebb \& Atherton, 2011). Ademais novos estudos, relacionaram o ritmo circadiano e a melatonina na patogênese de úlceras.

A melatonina (MT), formada enzimaticamente a partir do L-triptofano (TRP), é uma molécula hormonal liberada no lúmen do trato gastrointestinal (TGI) e absorvida na circulação portal, sendo metabolizada no fígado e liberada com bile no duodeno. A síntese da melatonina ocorre sob influência de duas enzimas principais: arilalquilamina- $\mathrm{N}$-acetiltransferase (AANAT) e hidroxiindol-O-metiltransferase (HIOMT), transformando o triptofano em melatonina (Konturek et al., 2007).

Na porção superior do TGI, a melatonina exibe um amplo espectro de atividades como sincronização circadiana por estímulos externos, atividade de eliminação de radicais livres, proteção da mucosa contra vários irritantes e cicatrização de várias lesões, como estomatite, esofagite, gastrite e úlcera péptica (Akinci, Esrefoglu, Cetin \& Ates, 2015; Brzozowski et al., 2007).

O tratamento de úlceras no TGI envolve diversas substâncias, como esquemas de inibidores de bomba de prótons (IBP) e antimicrobianos (claritromicina, furazolidona, amoxicilina, metronidazol, tetraciclina, bismuto e quinolonas) (Bittencourt et al., 2006; Ber Nieto et al., 2012). Entretanto, têm sido desenvolvidos estudos experimentais acerca da efetividade da melatonina e L-triptofano como substâncias gastroprotetoras, agindo contra lesões na mucosa do TGI (Konturek, Konturek \& Celinsky, 2010). MT ou TRP combinado com omeprazol acelerariam o processo de cicatrização de úlceras gastroduodenais de H. pylori, sendo esse efeito cicatrizante atribuído aos incrementos nos níveis plasmáticos de MT (Celinski, Konturek \& Konturek, 2011).

Um estudo desenvolvido por Celinski et al., em 2011, demonstrou pela primeira vez que MT exógena e aquela gerada no TGI contribuem para a cicatrização da úlcera gastroduodenal infectada por H. pylori, provavelmente devido aos efeitos antioxidantes e anti-inflamatórios da MT e da leptina produzidas em tecidos gástricos. No estudo desenvolvido por Abdelraheim et. al. (2015) realizando-se a administração de MT no tratamento de úlceras em ratos, observou-se diminuição significativa nos níveis de grelina e expressão de mRNA, bem como níveis reduzidos de glutationa (GSH) no grupo de ratos 
com úlcera em comparação com os controles. Os exames histológicos revelaram lesões graves da mucosa induzidas por etanol que foram significativamente melhoradas pela administração de melatonina.

A relação entre mecanismos cronobiológicos com a patogênese de úlceras é relativamente recente no âmbito da Medicina. Embora a maior parte dos trabalhos apontem as inúmeras vantagens da administração de melatonina para tratamento de lesões induzidas por úlceras, ainda são escassos os trabalhos que reúnam as comprovações científicas acerca dessa temática, embora seja de grande relevância para a inovação em tratamentos de úlceras.

Dessa forma, constitui-se proposta desse trabalho, analisar comparativamente resultados e relações acerca da melatonina como fator gastroprotetor e cicatrizante em úlceras gastroduodenais, além de elucidar acerca da influência da cronobiologia na patogênese de úlceras.

\section{Metodologia}

O presente estudo consiste em uma revisão integrativa de literatura de natureza qualitativa (Ludke \& Andre, 2013). A pesquisa foi desenvolvida por meio de seis etapas: (1) identificação da temática, definição do problema e pergunta norteadora; (2) busca pelas melhores evidências para responder a pergunta norteadora; (3) avaliação dos estudos pré-selecionados e selecionados; (4) integração das evidências; (5) discussão dos resultados baseados em evidências; (6) síntese do conhecimento produzido (Botelho; Cunha; Macedo; 2011).

A pergunta norteadora da pesquisa foi: "Quais os efeitos da melatonina como tratamento complementar de úlceras gastroduodenais?".

Uma busca ativa foi realizada nas bases de dados Scientific Eletronic Library Online (SciELO), PubMed, Science Direct e Literatura Latino Americana e do Caribe em Ciências da Saúde (LILACS). Foram ultilizados descritores do vocabulário Medical Subject Headings (MeSH): "ulcer” (úlcera), "chronobiology” (cronobiologia), "melatonine” (melatonina), "peptic ulcer" (úlcera péptica) e "duodenal ulcer" (úlcera duodenal), sendo estes combinados com os operadores booleanos "and" e "or". A busca foi realizada em agosto de 2020, sendo incluídos na revisão estudos quantitativos e qualitativos, artigos escritos em inglês, português, espanhol e francês. Cada referência foi importada para o Software EndNote, através do qual foram excluídas as repetições.

Os critérios de inclusão utilizados na seleção dos artigos foram: 1) estudos de língua inglesa, portuguesa, espanhola e francesa; 2) ensaios clínicos randomizados, caso-controle, coorte, transversal ou séries de casos. Os critérios de exclusão foram: 1) estudos prospectivos não randomizados; 2) estudos sem texto completo e 3) estudos que não atendiam ao objetivo do trabalho de explicar e comprovar a influência do uso terapêutico de melatonina em casos de úlceras gastroduodenais.

Após a aplicação dos critérios de elegibilidade e exclusão, os artigos escolhidos tiveram algumas informações principais extraídas. Foram retiradas as variáveis: título, autores, tipo de publicação, $\mathrm{n}^{\circ}$ de registro, título da revista, ano de publicação, volume, páginas, país, população a qual o estudo foi dirigido (homens, mulheres, homens e mulheres, in vitro, roedores ou primatas não humanos), as características da lesão, região da lesão ulcerativa, quais drogas foram utilizadas juntamente com a melatonina, tempo de tratamento e comorbidades presentes.

\section{Resultados e Discussão}

A busca nas bases de dados utilizando os descritores selecionados resultou em 540.364 artigos. Destes, após a avaliação dos títulos e resumos, foram removidos os que não atendiam à proposta do trabalho e aos critérios de inclusão, restando 76 artigos a serem analisado na íntegra, processo que resultou na seleção de 29 trabalhos (Figura 1). 
Os trabalhos selecionados consistiram em ensaios clínicos randomizados, caso-controle, coorte, transversal e séries de casos. Foram utilizados os trabalhos que traziam resultados da aplicação terapêutica de melatonina em lesões gastroduodenais ulcerativas.

Figura 1 - Representação esquemática dos métodos de identificação, triagem, elegibilidade e inclusão de trabalhos.

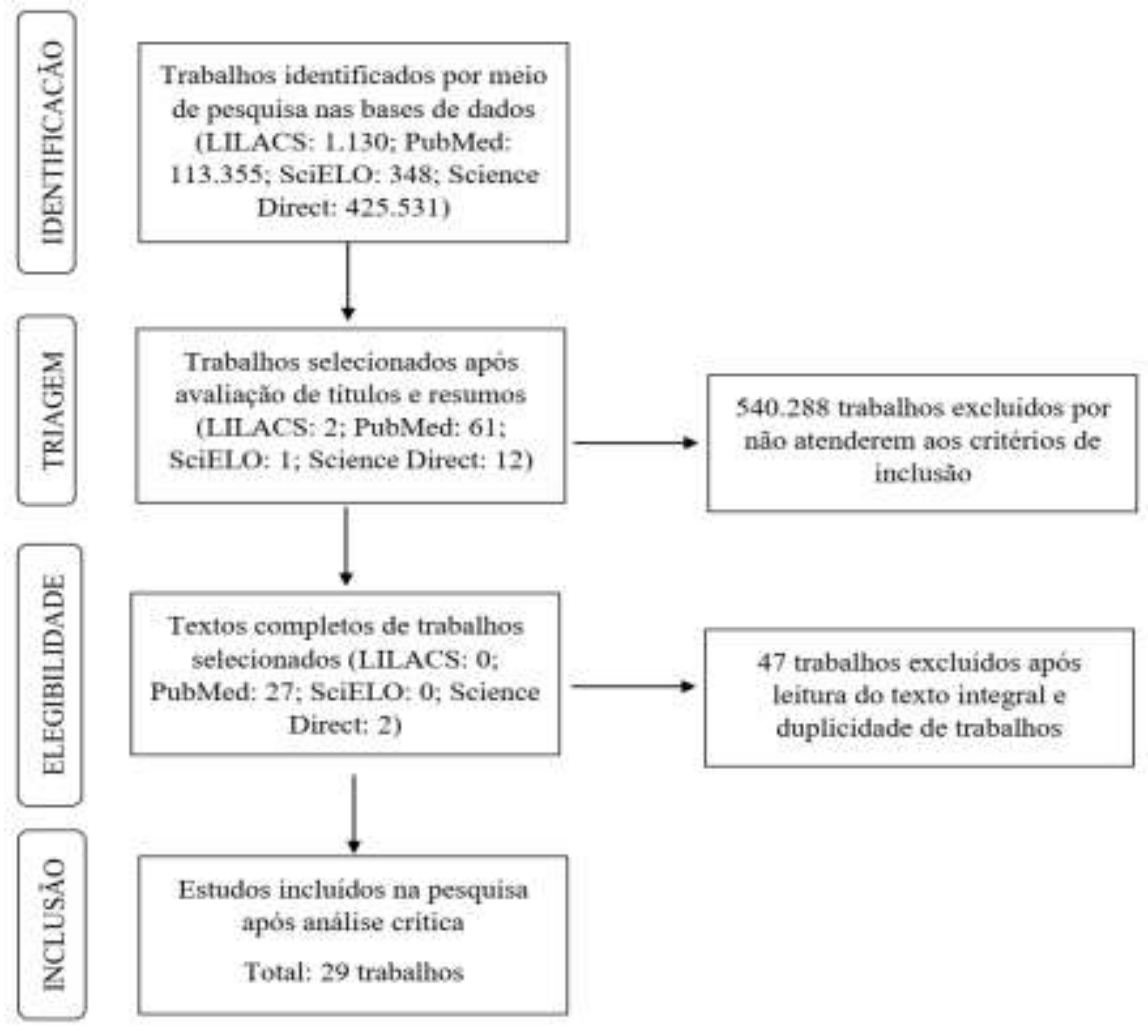

Fonte: Adaptada de acordo com o PRISMA Flow Diagram.

Em um formulário de dados padronizado, foram compiladas as seguintes informações dos estudos analisados: as características do estudo, como nome do autor, referência, ano de publicação, tamanho da amostra e população, medicamentos utilizados, metodologia e resultados. Os detalhes dos estudos estão relatados na tabela 1.

Tabela 1 - Estudos utilizando melatonina no tratamento de úlceras gastroduodenais.

\begin{tabular}{|c|c|c|c|c|}
\hline Autoria & $\begin{array}{l}\text { Tipo de estudo e } \\
\text { população }\end{array}$ & Medicamentos & Objetivo & Resultados \\
\hline $\begin{array}{l}\text { Akinci et al. } \\
\qquad(2015)\end{array}$ & $\begin{array}{l}\text { Estudo comparativo; } \\
\text { ratos machos }(n=56)\end{array}$ & $\begin{array}{l}\text { Melatonina, ácido } \\
\text { ascórbico e beta- } \\
\text { caroteno }\end{array}$ & $\begin{array}{l}\text { Comparação do efeito da melatonina } \\
\text { com outros antioxidantes na lesão } \\
\text { gástrica induzida }\end{array}$ & $\begin{array}{l}\text { A melatonina evitou mudanças } \\
\text { histológicas }\end{array}$ \\
\hline $\begin{array}{l}\text { Arabski et al. } \\
\quad(2006)\end{array}$ & $\begin{array}{l}\text { Ensaio clínico; } \\
\text { homens e mulheres } \\
\qquad(\mathrm{n}=40)\end{array}$ & $\begin{array}{l}\text { Vitamina } \mathrm{C} \text { e } \mathrm{E}, \\
\text { quercetina e melatonina }\end{array}$ & $\begin{array}{l}\text { Comparação do efeito da melatonina } \\
\text { com outros antioxidantes na lesão } \\
\text { gástrica induzida }\end{array}$ & $\begin{array}{l}\text { Melatonina apresentou maior redução } \\
\text { nos danos celulares }\end{array}$ \\
\hline $\begin{array}{l}\text { Bandyopadhyay } \\
\text { et al. (2000) }\end{array}$ & Estudo caso-controle & $\begin{array}{l}\text { Pré-tratamento com } \\
\text { melatonina, omeprazol, }\end{array}$ & $\begin{array}{lccr}\text { Comparações } & \text { dos } & \text { efeitos } & \text { da } \\
\text { melatonina } & \text { com } & \text { ranitidina } & \text { e }\end{array}$ & $\begin{array}{l}\text { Melatonina diminui o dano gástrico } \\
\text { induzido por estresse e indometacina, }\end{array}$ \\
\hline
\end{tabular}




\begin{tabular}{|c|c|c|c|c|}
\hline & com ratos e in vitro & $\begin{array}{l}\text { ranitidina, vitamina } \mathrm{E}, \\
\text { vitamina } \mathrm{C} \text { e glutationa }\end{array}$ & $\begin{array}{l}\text { omeprazol e outros antioxidantes; } \\
\text { além da ação da melatonina sob o } \\
\text { radical hidroxila in vitro }\end{array}$ & $\begin{array}{l}\text { reduziu diretamente os } \\
\text { hidroxila in vitro }\end{array}$ \\
\hline $\begin{array}{l}\text { Bandyopadhyay } \\
\text { et al. (2004) }\end{array}$ & $\begin{array}{l}\text { Estudo comparativo; } \\
\text { ratos }(\mathrm{n}=30)\end{array}$ & Piroxicam e melatonina & $\begin{array}{l}\text { Estudo sobre o efeito da melatonina } \\
\text { em úlceras induzidas por Piroxicam }\end{array}$ & $\begin{array}{l}\text { A melatonina reduziu os danos gástricos } \\
\text { induzidos por piroxicam e indometacina }\end{array}$ \\
\hline $\begin{array}{l}\text { Bandyopadhyay } \\
\text { et al. (2002) }\end{array}$ & $\begin{array}{l}\text { Estudo caso-controle } \\
\text { com ratos }(\mathrm{n}=30) \text { e in } \\
\text { vitro }\end{array}$ & $\begin{array}{l}\text { Melatonina, ranitidina } \\
\text { e omeprazol }\end{array}$ & $\begin{array}{l}\text { Observação do efeito antiúlcera da } \\
\text { melatonina nas lesões gástricas } \\
\text { causadas pelo estresse por frio, } \\
\text { comparando a ranitidina e omeprazol }\end{array}$ & $\begin{array}{l}\text { A melatonina evitou danos gástricos } \\
\text { induzidos, eliminou radicais hidroxilas } \\
\text { gerados durante condições de estresse in } \\
\text { vivo e in vitro, diminuiu a atividade da } \\
\text { peroxidase gástrica e aumentou a } \\
\text { atividade da superóxido dismutase } \\
\text { mitocondrial gástrica }\end{array}$ \\
\hline $\begin{array}{l}\text { Bilici et al. } \\
\quad \text { (2002) }\end{array}$ & $\begin{array}{l}\text { Estudo controlado; } \\
\text { ratos machos }(n=32)\end{array}$ & $\begin{array}{l}\text { Pré-tratamento com } \\
\text { melatonina } \\
\text { omeprazol }\end{array}$ & $\begin{array}{l}\text { Estudo sobre o efeito da melatonina } \\
\text { em úlceras induzidas por etanol }\end{array}$ & $\begin{array}{l}\text { A melatonina aumentou tanto a } \\
\text { concentração de glutationa total (tGSH) } \\
\text { quanto a atividade de glutationa } \\
\text { redutase (GSSG-Rd). Também, reduziu } \\
\text { a infiltração de leucócitos } \\
\text { polimorfonucleares induzida por etanol } \\
\text { no estômago e a extensão da lesão }\end{array}$ \\
\hline $\begin{array}{l}\text { Brzozowski et al. } \\
\text { (1997) }\end{array}$ & $\begin{array}{l}\text { Estudo caso-controle; } \\
\text { ratos ambos os sexos }\end{array}$ & $\begin{array}{lr}\text { Melatonina, } & \text { L- } \\
\text { triptofano } & \mathrm{e} \\
\text { indometacina } & \end{array}$ & $\begin{array}{l}\text { Observação dos efeitos da melatonina } \\
\text { e do L-triptofano em lesões gástricas } \\
\text { induzidas por etanol, estresse, aspirina } \\
\text { e isquemia-perfusão }\end{array}$ & $\begin{array}{l}\text { Melatonina e L-triptofano não afetaram } \\
\text { as lesões gástricas induzidas por etanol } \\
\text { e aspirina, mas, nos casos estresse e } \\
\text { isquemia/perfusão, houve redução das } \\
\text { lesões }\end{array}$ \\
\hline $\begin{array}{l}\text { Brzozowski et al. } \\
\text { (2005) }\end{array}$ & $\begin{array}{l}\text { Estudo comparativo; } \\
\quad \text { ratos machos }\end{array}$ & $\begin{array}{l}\text { Melatonina e L- } \\
\text { triptofano, luzindole e } \\
\text { indometacina }\end{array}$ & $\begin{array}{l}\text { Estudo sobre o efeito da melatonina } \\
\text { em úlceras induzidas por anti- } \\
\text { inflamatórios sob administração de } \\
\text { luzindole }\end{array}$ & $\begin{array}{l}\text { Os efeitos gastroprotetores da } \\
\text { melatonina, mediados pelos seus } \\
\text { receptores, como o MT2, podem ser } \\
\text { atenuados pelo luzindole }\end{array}$ \\
\hline $\begin{array}{l}\text { Celinski et al. } \\
\qquad(2011)\end{array}$ & $\begin{array}{l}\text { Ensaio clínico; } \\
\text { homens e mulheres } \\
\qquad(\mathrm{n}=24)\end{array}$ & $\begin{array}{l}\text { Omeprazol, placebo e } \\
\text { melatonina }\end{array}$ & $\begin{array}{l}\text { Avaliar a eficácia do MT e TRP } \\
\text { adicionado ao omeprazol nas úlceras } \\
\text { gástricas ou duodenais comuns ao } \mathrm{H} \text {. } \\
\text { pylori positivas }\end{array}$ & $\begin{array}{l}\text { A melatonina exógena contribuiu para a } \\
\text { cicatrização de úlcera gastroduodenal } \\
\text { infectada por H. pylori }\end{array}$ \\
\hline $\begin{array}{l}\text { Celinski et al. } \\
\qquad(2011)\end{array}$ & $\begin{array}{l}\text { Ensaio clínico; } \\
\text { homens e mulheres } \\
\qquad(\mathrm{n}=28)\end{array}$ & $\begin{array}{l}\text { Omeprazol, placebo, } \\
\text { melatonina e triptofano }\end{array}$ & $\begin{array}{l}\text { Avaliar os efeitos da melatonina ou de } \\
\text { seu precursor TRP, em combinação } \\
\text { com omeprazol }\end{array}$ & $\begin{array}{l}\text { A melatonina ou seu precursor TRP } \\
\text { aplicado por via oral são eficazes para } \\
\text { acelerar a taxa de cicatrização da úlcera }\end{array}$ \\
\hline Cho et al. (1989) & $\begin{array}{l}\text { Estudo comparativos; } \\
\text { ratos machos }\end{array}$ & $\begin{array}{l}\text { Serotonina } \\
\text { melatonina }\end{array}$ & $\begin{array}{l}\text { Análise dos efeitos da melatonina e da } \\
\text { serotonina diante de alterações fluxo } \\
\text { sanguíneo da mucosa gástrica e de } \\
\text { lesões hemorrágicas causadas por } \\
\text { etanol }\end{array}$ & $\begin{array}{l}\text { O pré-tratamento com melatonina } \\
\text { evitou a formação de lesões gástricas } \\
\text { induzidas por etanol mas não impediu a } \\
\text { redução do fluxo sanguíneo }\end{array}$ \\
\hline $\begin{array}{l}\text { De La Lastra et } \\
\text { al. (1997) }\end{array}$ & $\begin{array}{l}\text { Estudo comparativo; } \\
\text { ratos de ambos os } \\
\text { sexos }\end{array}$ & Melatonina & $\begin{array}{l}\text { Examinar os efeitos protetores in vivo } \\
\text { da melatonina no dano gástrico } \\
\text { induzido por isquemia-reperfusão }\end{array}$ & $\begin{array}{l}\text { A administração intraperitoneal de } \\
\text { melatonina evitou a lesão pós-isquêmica } \\
\text { da mucosa }\end{array}$ \\
\hline De La Lastra et & Estudo comparativo; & Indometacina & Estudo sobre o efeito da melatonina & melatonina \\
\hline
\end{tabular}


Research, Society and Development, v. 10, n. 6, e52310616087, 2021

(CC BY 4.0) | ISSN 2525-3409 | DOI: http://dx.doi.org/10.33448/rsd-v10i6.16087

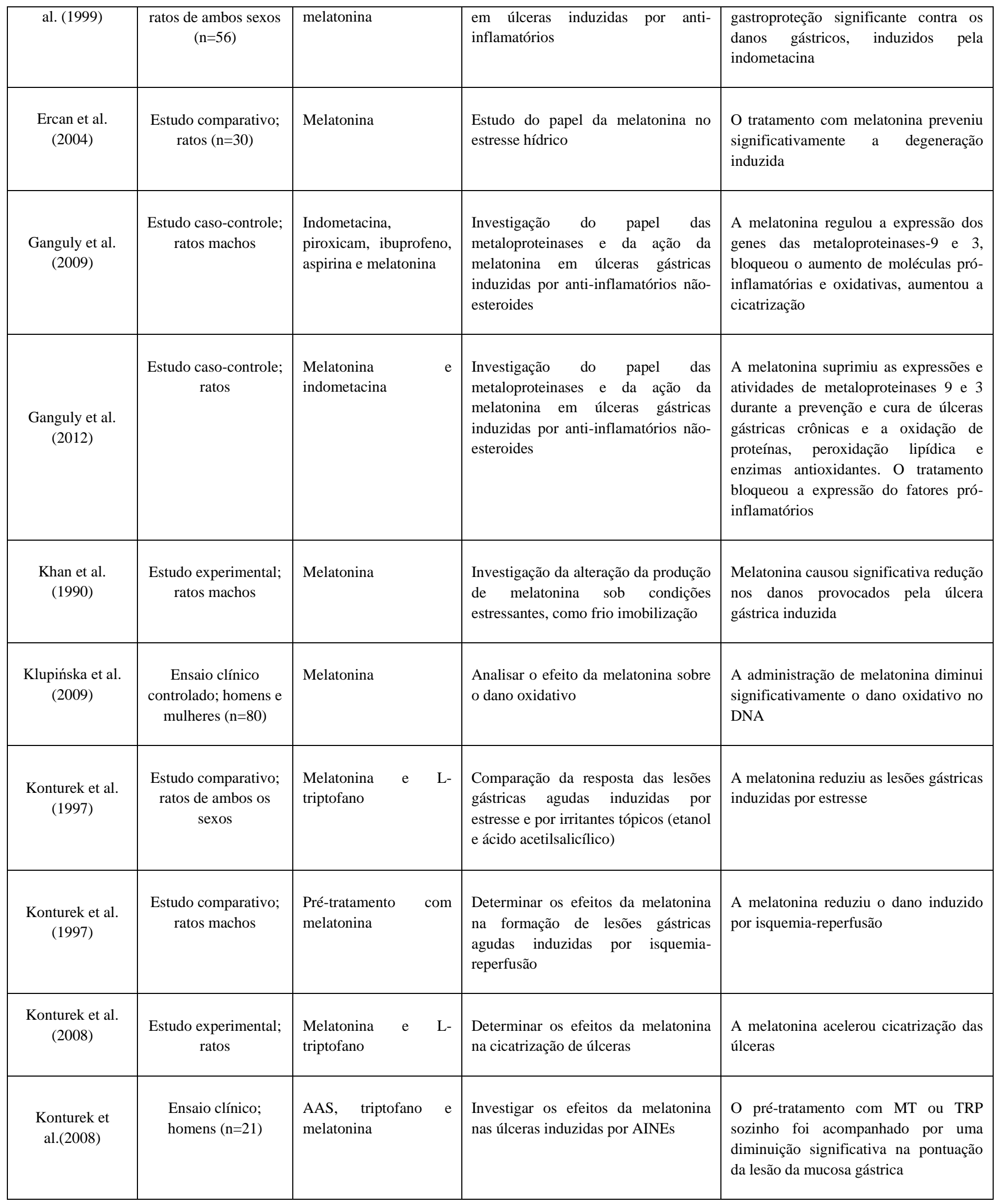




\begin{tabular}{|c|c|c|c|c|}
\hline Lin et al. (2020) & $\begin{array}{l}\text { Estudo caso-controle; } \\
\text { camundongos } \\
\text { machos }(\mathrm{n}=144)\end{array}$ & $\begin{array}{l}\text { Pre-tratamento com } \\
\text { melatonina }\end{array}$ & $\begin{array}{l}\text { Investigar os potenciais efeitos da } \\
\text { melatonina como pré-tratamento a } \\
\text { úlceras por estresse }\end{array}$ & $\begin{array}{l}\text { A melatonina reduziu a lesão } \\
\text { intestinal induzida por estresse }\end{array}$ \\
\hline Luo et al.(2018) & $\begin{array}{l}\text { Estudo comparativo; } \\
\text { camundongos fêmeas }\end{array}$ & $\begin{array}{l}\text { Suspensão oral de } \mathrm{H} \text {. } \\
\text { pylori e melatonina }\end{array}$ & $\begin{array}{l}\text { Observação dos mecanismos } \\
\text { genéticos e efeitos da melatonina em } \\
\text { gastrite induzidas por H. pylori }\end{array}$ & $\begin{array}{l}\text { A melatonina promove proteção } \\
\text { gástrica por meio da supressão dos } \\
\text { receptores toll like } 2 \text { e ativação dos } \\
\text { receptores toll like } 4\end{array}$ \\
\hline $\begin{array}{l}\text { Mohamadin et al. } \\
\text { (2009) }\end{array}$ & $\begin{array}{l}\text { Estudo experimental; } \\
\text { ratos machos }(n=30)\end{array}$ & $\begin{array}{l}\text { Peróxido de hidrogênio } \\
\text { e melatonina }\end{array}$ & $\begin{array}{l}\text { Investigar os potenciais efeitos da } \\
\text { melatonina na indução de danos na } \\
\text { mucosa gástrica. }\end{array}$ & $\begin{array}{l}\text { Melatonina reduziu os danos na mucosa } \\
\text { gástrica contra o peróxido de hidrogênio } \\
\text { e possibilitou um maior fluxo sanguíneo } \\
\text { nessa região }\end{array}$ \\
\hline $\begin{array}{l}\text { Sener-Muratoğlu } \\
\text { et al.(2001) }\end{array}$ & $\begin{array}{l}\text { Estudo comparativo; } \\
\text { ratos de ambos os } \\
\text { sexos }\end{array}$ & $\begin{array}{l}\text { Omeprazol, famotidina, } \\
\text { melatonina e ácido } \\
\text { acetilsaclicílico (AAS) }\end{array}$ & $\begin{array}{l}\text { Observação do efeito da melatonina } \\
\text { sobre úlcera gástrica induzida por } \\
\text { AAS }\end{array}$ & $\begin{array}{l}\text { Famotidina e omeprazol diminuíram a } \\
\text { acidez gástrica, que foi aumentada pelo } \\
\text { AAS, enquanto a melatonina não teve } \\
\text { efeito sobre este parâmetro }\end{array}$ \\
\hline $\begin{array}{l}\text { Zhang et al. } \\
\text { (2015) }\end{array}$ & $\begin{array}{l}\text { Estudo comparativo; } \\
\text { ratos machos }(\mathrm{n}=40)\end{array}$ & Melatonina & $\begin{array}{l}\text { Observação do efeito da melatonina } \\
\text { sobre úlcera gástrica induzida por } \\
\text { estresse sonoro }\end{array}$ & $\begin{array}{l}\text { A melatonina melhorou o exame } \\
\text { patológico, a pontuação de lesão de } \\
\text { Guth, níveis de cortisol, motilina, a } \\
\text { atividade da superóxido dismutase e } \\
\text { malondialdeído }\end{array}$ \\
\hline
\end{tabular}

Fonte: Autores.

\subsection{Fisiologia da Melatonina}

A melatonina ou N-acetil-5-metoxitriptamina é um hormônio sintetizado por várias células e tecidos, presente em qualquer compartimento do organismo por causa de sua característica anfifílica (Amaral \& Cipolla-Neto, 2018). A síntese central de melatonina se dá pela glândula pineal, obedecendo um padrão rítmico circadiano, sincronizado pelos núcleos supraquiasmáticos localizados no hipotálamo, sendo esses núcleos regulados pelo ciclo claro-escuro ambiental através do trato retinohipotalâmico (Cipolla-Neto \& Amaral, 2018). Nesse sentido, a síntese e degradação da melatonina sofre influência de estímulos luminosos, principalmente na faixa azul, que ativam a degradação da melanopsina nas células ganglionares fotorreceptivas da retina que se projetam, pelo trato retinohipotalâmico, até os núcleos supraquiasmáticos, inibindo sua síntese (Cipolla-Neto \& Amaral, 2018). 
Além disso, as células da glândula pineal produtoras de melatonina, os pinealócitos, estão sob o controle de um sistema neural que inclui os núcleos paraventriculares do hipotálamo, a medula espinal e o sistema nervoso autônomo. Quando os terminais simpáticos liberam norepinefrina, esta é captada pelos receptores noradrenérgicos beta e alfa na membrana dos pinealócitos, ocorrendo a ativação das vias cAMP-PKA-CREB e PLC-Ca ${ }^{++}$-PKC para desencadear a síntese de melatonina (Vollrath et al., 1981; Kappers et al., 1960).

A síntese da melatonina se inicia a partir do triptofano transformado em 5-hidroxitriptofano em uma reação química catalisada pela triptofano hidroxilase. O 5-hidroxitriptofano é convertido em serotonina, a qual é acetilada, pela arilalquilamina $\mathrm{N}$-acetiltransferase em $\mathrm{N}$-acetilserotonina que é convertida em melatonina pela acetilserotonina O-metiltransferase (ASMT), anteriormente chamada de hidroxi-indol-O-metiltransferase (HIOMT) (Cipolla-Neto \& Amaral, 2018; Afeche et al., 2008; Reiter et al., 1991).

A vascularização e fixação da glândula pineal na parede do terceiro ventrículo permite que a melatonina seja liberada no líquido cefalorraquidiano durante a noite, bem como na corrente sanguínea. A melatonina é transportada no sangue ligada à albumina, e é metabolizada por duas vias: no fígado e no sistema nervoso central. No fígado é metabolizada em 6hidroximelatonina e conjugada a 6-sulfatoximelatonina para a posterior excreção urinária. No sistema nervoso central, a melatonina é degradada em N-acetil-N2-formil-5-metoxiquinuramina (Maganhin et al., 2008).

Assim como mencionado anteriormente, existem algumas fontes extrapineais, dentre elas está o trato gastrointestinal. Segundo Bubenik et al. (2002), a concentração de melatonina nos tecidos gastrointestinais ultrapassa os níveis sanguíneos em 10-100 vezes e há pelo menos 400 vezes do que na pineal glândula. No trato gastrointestinal, a melatonina desempenha diversas funções, dentre elas: (1) regeneração e função do epitélio; (2) aumenta o sistema imunológico do intestino; (3) reduz o tônus dos músculos gastrointestinais; (4) ação antioxidante; (5) redução da excreção de ácido clorídrico e (6) aumenta a microcirculação (Reiter et al., 1991; Bubenik et al., 2002; Terry et al., 2009; Konturek, Brzozwski \& Konturek, 2011; Ferreira et al., 2010; Reiter et al., 2016).

\subsection{Patogênese e Tratamento de Úlceras Gastroduodenais}

Embora as úlceras gastroduodenais sejam reconhecidas desde o período da antiguidade, grandes avanços têm ocorrido desde o final do século XIX, modificando o entendimento acerca desta condição clínica e possibilitando novas alternativas de tratamento. A úlcera péptica consiste em uma lesão no revestimento interno do estômago, duodeno ou esôfago, com etiologias multifatoriais e muitas vezes desconhecidas, resultando em ruptura da mucosa e atingindo a submucosa. As etiologias mais comuns consistem em: (1) infecção por H. pylori; (2) uso excessivo de anti-inflamatórios; (3) fatores dietéticos; (4) estresse; (5) neoplasias; (6) infecções. As causas mais raras constituem as doenças granulomatosas, síndrome de Zollinger-Ellison, hiperpatiroidismo e Tecido pancreático ectópico. A infecção gástrica pela bactéria Helicobacter pylori, sendo responsável por mais de $95 \%$ dos casos de úlcera duodenal e $80 \%$ dos portadores de úlcera gástrica (Reiter et al., 2016; Mégraud et al., 1993; Harris \& Misiewicz, 2001).

A fisiopatologia das úlceras pépticas remete à existência de agressores que promovem um estresse à mucosa digestiva, seja por um aumento na acidez, liberação de pepsina, distúrbios de motilidade ou sistema de defesa da mucosa prejudicado, fatores esses desenvolvidos por inúmeras etiologias (Holle et al., 1985).

O controle da secreção ácida é mediado por fatores endócrinos (hormônio gastrina), nervoso (nervo vago) e substâncias parácrinas (histamina). Após a alimentação, os níveis de acidez desencadeando maior secreção de pepsina pelas células parietais do estômago que estimulam os nervos vagos. Em paciente com úlceras, ocorrem uma série de anormalidades na secreção de ácido. Indivíduos infectados por H. pylori apresentam respostas elevadas à gastrina, havendo uma secreção de ácido mais elevada que o normal (Mertz \& Walsh, 1991). 
As úlceras pépticas também estão associadas a um aumento no pepsinogênio 1, sendo um marcador da úlcera péptica (Samloff, Varis \& Ihamaki, 1992). A infecção por H. pylori desencadeia aumento nos níveis de pepsina por interferir na liberação de pepsinogênio 1 (Oderda, Vaira \& Holton, 1982). A motilidade e o tônus muscular gastrointestinal também são variáveis envolvidas nas úlceras, por estarem relacionada a um esvaziamento gástrico rápido. As mudanças no muco que protege o estômago e duodeno corroboram com uma menor proteção da camada mucosa. Pacientes com úlcera possuem muco com menos dextranos e glicolipoproteínas devido a maiores concentrações de endopeptidases que resultam na diminuição da viscosidade (Yunan et al., 1982; Slomiany, Bilski \& Sarosiek, 1987). Distúrbios na secreção de bicarbonato também interferem na maior probabilidade de desenvolver úlceras (Wallace et al., 1990).

A microcirculação e o fluxo sanguíneo são essenciais para a integridade dos tecidos, estando relacionados a uma maior responsividade às mudanças do sistema gastrointestinal (Pihan et al., 1986). Os anti-inflamatórios não esteroidais podem desencadear lesões agudas da mucosa, por ulcerações e hemorragias (Kurata et al, 1990). As úlceras também possuem forte componente psicossocial, assim como registrado durante a II Guerra Mundial os casos de úlcera péptica tornaram-se duas vezes mais elevados (Rezende et al., 2009).

O tratamento de úlceras pépticas comumente consiste no bloqueio da acidez gástrica. Os inibidores de bombas de prótons (IBPs) ou de citrato de ranitidina bismuto (RBC) são as medicações de escolha, combatendo a hiperacidez gástrica (Malfertheiner et al., 2002; Coelho et al., 2000). Os bloqueadores de histamina-2 (H2) também inibem a produção de ácido de maneira eficaz (Moraes et al., 1999). Os procedimentos cirúrgicos são raramente necessários, apenas quando as úlceras tornamse complicadas. Além de neutralizadores do ácido gástrico e tratamento de H. Pylori (Carretero et al., 2016).

\subsection{Possíveis Mecanismos de Ação da Melatonina no Tratamento de Úlceras Gastroduodenais}

No trato gastrointestinal, a melatonina é liberada e sintetizada por células enterocromáticas, obedecendo um padrão circadiano e sob ação de fatores nutricionais. A melatonina é distribuída de maneira heterogênea no sistema digestivo, nas camadas mucosa, submucosa e muscular do esôfago, na camada glandular da parede gástrica e nas criptas de Lieberkühn e glândulas de Brunner do duodeno, locais de desenvolvimento das úlceras pépticas (Bubenik et al., 1977). A melatonina colabora de diversas maneiras na capacidade defensiva da mucosa do sistema gastrointestinal, interferindo na permeabilidade da barreira muco-bicarbonato, capacidade de restituição e regeneração da mucosa, fluxo sanguíneo, colaborando com a ação das prostaglandinas ou sistema nervoso sensorial, entre outros (Motilva et al., 2001).

A melatonina pode atuar tanto na remediação quanto na prevenção das úlceras. A administração de melatonina via oral foi eficaz para acelerar a taxa de cicatrização da úlcera por suas atividades antioxidante e anti-inflamatórias no ensaio de Celinski et al. (2011). Ganguly et al. (2012) constataram o papel da melatonina tanto no tratamento das úlceras existentes como na prevenção, sendo observado que a melatonina suprimiu as expressões e atividades das metaloproteinases MMP-9 e -3 durante a prevenção e cura de úlceras gástricas crônicas, além de atuar na oxidação de proteínas, na peroxidação lipídica e atividade das enzimas antioxidantes. O tratamento bloqueou a expressão do fator de necrose tumoral (TNF) - $\alpha$, interleucina (IL) $-1 \beta$ e IL-8, reverteu a fosforilação elevada da quinase regulada extracelular (ERK) $1 / 2$ e da quinase N-terminal c-Jun

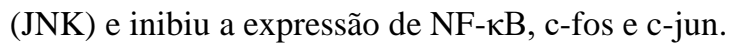

Em outros estudos também foi possível observar que a melatonina possui atividade contra o metabolismo oxidativo nas úlceras agudas induzidas por isquemia-reperfusão, com uma consequente melhora nas lesões e induzindo um aumento na atividade das enzimas antioxidantes (De La Lastra et al., 1997; Barlow-Balden et al., 1995). No que diz respeito às úlceras induzidas por medicamentos, os AINEs tem sido reconhecidos por sua ação gastrointestinal tóxica, seja pela inibição da síntese de prostaglandinas protetoras, lesões vasculares ou mudanças apoptóticas no epitélio gástrico. Nesse sentido, dados apontam o efeito da melatonina como gastroprotetor em casos de úlceras induzidas por anti-inflamatórios. O pré-tratamento com 
melatonina foi acompanhado em diversos ensaios por uma diminuição significativa na pontuação das lesões da mucosa gástrica induzidas por aspirina (Konturek et al., 2008).

O efeito antiúlcera da melatonina nas lesões gástricas causadas pelo piroxicam foi também analisado por Bandyopadhyay et al. (2004), em que foi constatado que a melatonina foi capaz de reduzir os danos gástricos induzidos tanto por piroxicam como de indometacina de maneira dependente da dose, com mais de $90 \%$ de inibição em uma dose de $60 \mathrm{mg} / \mathrm{kg}$ de peso corporal. Com o pré-tratamento em ratos por melatonina foi possível manter estáveis os níveis da peroxidação lipídica, oxidação de proteínas e do conteúdo de glutationa do tecido gástrico, os quais costumam aumentar com essas medicações.

Além disso, lesões gástricas subjacentes induzidas por estresse foram extensivamente investigadas também. O etanol provoca danos na mucosa gastrointestinal, por esfoliação celular e erosão, com consequente aumento em infiltrado inflamatório, espécies oxidativas de oxigênio e produtos vasoativos de mastócitos, macrófagos e outras células sanguíneas, causando necrose e úlceras (Szabó et al., 1987). O tratamento com melatonina reduziu o grau de infiltração de leucócitos e também aumentou a concentração de glutationa reduzida e a atividade das enzimas glutationa redutase e glutationa peroxidase, nas lesões induzidas por etanol (Motilva et al., 2001). No estudo experimental em ratos de Cho et al. (1989), a pré-incubação com melatonina reverteu significativamente as alterações vasculares e aliviou a gravidade da ulceração por etanol.

\section{Considerações Finais}

A melatonina além de estar relacionada à função reguladora do ciclo circadiano, está fortemente associada à diminuição da atividade inflamatória e atua na modulação imunológica e gastrointestinal, podendo atuar tanto na remediação quanto na prevenção das úlceras. A administração de melatonina mostrou-se eficaz e segura para acelerar a taxa de cicatrização das úlceras gastroduodenais de distintas etiologias por suas atividades antioxidante e anti-inflamatórias por diversos fatores, dentre eles: a regeneração e função da mucosa; aumento da atividade do sistema imunológico; redução do tônus dos músculos gastrointestinais; ação antioxidante; redução da excreção de ácido clorídrico e aumento do fluxo sanguíneo. Entretanto, a literatura é ainda escassa quanto à realização do tratamento ou pré-tratamento das úlceras com melatonina, seja isoladamente ou em conjunto com outras drogas. Portanto, é sugerido para trabalhos futuros a realização de maiores investigações acerca da temática com população de seres humanos.

\section{Referências}

Abdelraheim, S. R., Okasha, A. M., Ghany, H. M., \& Ibrahim, H. M. (2015). Ghrelin gene expression in rats with ethanol-induced gastric ulcers: a role of melatonin. Endocrine regulations, 49(1), 3-10.

Afeche S.C., do Amaral F.G., Villela D.C.M., Abrahão M.V., Peres R., Cipolla-Neto J. (2008). Melatonin and the Pineal Gland. In: Romano E, De Luca S, editors. New Research on Neurosecretory Systems. New York: Nova Biomedical Books; p. 151-77.

Akinci A., Esrefoglu M., Cetin A., Ates B. (2015). Melatonin is more effective than ascorbic acid and $\beta$-carotene in improvement of gastric mucosal damage induced by intensive stress. Arch Med Sci, 11 (5), 1129-36.

Alarcón de la Lastra, C., Motilva, V., Martín, M. J., Nieto, A., Barranco, M. D., Cabeza, J., \& Herrerías, J. M. (1999). Protective effect of melatonin on indomethacin-induced gastric injury in rats. Journal of pineal research, 26(2), 101-107.

Amaral FGD, Cipolla-Neto J (2018). A brief review about melatonin, a pineal hormone. Arch Endocrinol Metab, 62 (4), $472-9$.

Arabski, M., Kazmierczak, P., Wiśniewska-Jarosińska, M., Morawiec, Z., Morawiec-Bajda, A., Klupińska, G., Drzewoski, J., Chojnacki, J., \& Błasiak, J. (2006). Helicobacter pylori infection can modulate the susceptibility of gastric mucosa cells to MNNG. Cellular \& molecular biology letters, 11(4), 570-578.

Bandyopadhyay, D., Biswas, K., Bandyopadhyay, U., Reiter, R. J., \& Banerjee, R. K. (2000). Melatonin protects against stress-induced gastric lesions by scavenging the hydroxyl radical. Journal of Pineal Research, 29(3), 143-151.

Bandyopadhyay, D., Ghosh, G., Bandyopadhyay, A., \& Reiter, R. J. (2004). Melatonin protects against piroxicam-induced gastric ulceration. Journal of pineal research, 36(3), 195-203. 
Bandyopadhyay, D., Bandyopadhyay, A., Das, P. K., \& Reiter, R. J. (2002). Melatonin protects against gastric ulceration and increases the efficacy of ranitidine and omeprazole in reducing gastric damage. Journal of pineal research, 33(1), 1-7. https://doi.org/10.1034/j.1600-079x.2002.01107.x

Barlow-Walden, L., Reiter, R., Abe, M., Pablos, M., Menendez-Pelaez, A., Chen, L. D., \& Poeggeler, B. (1995). Melatonin stimulates brain glutathione peroxidase activity. Neurochemistry International, 26(5), 497-502.

Ber N.Y. et al. (2012). Protocolo Terapêutico de la Úlcera Péptica. Medicine - Programa de Formacíon Médica Continuada acreditado, 11 (3), 179-82.

Bilici, D., Süleyman, H., Banoğlu, Z. N., Kiziltunç, A., Avci, B., Ciftçioğlu, A., \& Bilici, S. (2002). Melatonin prevents ethanol-induced gastric mucosal damage possibly due to its antioxidant effect. Digestive diseases and sciences, 47(4), 856-861. https://doi.org/10.1023/a:1014764705864

Bittencourt P.F.S., Rocha G.A., Penna F.J., Queiroz D.M.M (2006). Úlcera péptica gastroduodenal e infecção pelo Helicobacter pylori na criança e adolescente. J. Pediatr, 82 (5), 325-334.

Botelho, L.L.R.; Cunha, C.C.A.; Macedo, M (2011). O método da revisão integrativa nos estudos organizacionais. Gestão e Sociedade: Belo Horizonte, v.5, n. 11, p. 121-136.

Brzozowski, T., Konturek, P. C., Konturek, S. J., Pajdo, R., Bielanski, W., Brzozowska, I., ... Hahn, E. G. (1997). The role of melatonin and L-tryptophan in prevention of acute gastric lesions induced by stress, ethanol, ischemia, and aspirin. Journal of Pineal Research, 23(2), 79-89.

Brzozowski, T., Konturek, P. C., Zwirska-Korczala, K., Konturek, S. J., Brzozowska, I., Drozdowicz, D., ... Hahn, E. G. (2005). Importance of the pineal gland, endogenous prostaglandins and sensory nerves in the gastroprotective actions of central and peripheral melatonin against stress-induced damage. Journal of Pineal Research, 39(4), 375-385.

Brzozowski T., Zwirska-Korczala K., Konturek P.C., Konturek S.J., Sliwowski Z., Pawlik M., et al (2007). Role of circadian rhythm and endogenous melatonin in pathogenesis of acute gastric bleeding erosions induced by stress. J Physiol Pharmacol, 58 (6), 53-64.

Bubenik GA (2002). Gastrointestinal melatonin: localization, function, and clinical relevance. Dig Dis Sci., 47(10):2336-48.

Bubenik, G. A., Brown, G. M., \& Grota, L. J. (1977). Immunohistological localization of melatonin in the rat digestive system. Experientia, 33(5), 662-663. Carretero, C. (2016). Úlcera péptica. Medicine - Programa de Formación Médica Continuada Acreditado, 12(3), 111-117.

Celinski K, Konturek P.C., Konturek S.J., Slomka M., Cichoz-Lach H., Brzozowski T., et al (2011). Effects of melatonin and tryptophan on healing of gastric and duodenal ulcers with Helicobacter pylori infection in humans. J Physiol Pharmacol, 62(5), 521-6.

Celinski K, Konturek SJ, Konturek PC, et al (2011). Melatonin or L-tryptophan accelerates healing of gastroduodenal ulcers in patients treated with omeprazole. J Pineal Res, 50, 389-394.

Cho, C. H., Pang, S. F., Chen, B. W., \& Pfeiffer, C. J. (1989). Modulating action of melatonin on serotonin-induced aggravation of ethanol ulceration and changes of mucosal blood flow in rat stomachs. Journal of pineal research, 6(1), 89-97.

Cipolla-Neto, J., \& Amaral, F. G. D. (2018). Melatonin as a Hormone: New Physiological and Clinical Insights. Endocrine Reviews, 39(6), 990-1028.

De La Lastra, C. A., Cabeza, J., Motilva, V., \& Martin, M. J. (1997). Melatonin protects against gastric ischemia-reperfusion injury in rats. Journal of pineal research, 23(2), 47-52.

Ercan, F., Cetinel, S., Contuk, G., Cikler, E., \& Sener, G. (2004). Role of melatonin in reducing water avoidance stress-induced degeneration of the gastrointestinal mucosa. Journal of pineal research, 37(2), 113-121.

Ferreira, C. D. S., Maganhin, C. C., Simões, R. D. S., Girão, M. J. B. C., Baracat, E. C., \& Soares-Jr, J. M. (2010). Melatonina: modulador de morte celular. Revista Da Associação Médica Brasileira, 56(6), 715-718.

Ganguly, K., \& Swarnakar, S. (2009). Induction of matrix metalloproteinase-9 and -3 in nonsteroidal anti-inflammatory drug-induced acute gastric ulcers in mice: regulation by melatonin. Journal of Pineal Research, 47(1), 43-55.

Ganguly, K., \& Swarnakar, S. (2012). Chronic gastric ulceration causes matrix metalloproteinases-9 and -3 augmentation: alleviation by melatonin. Biochimie, 94(12), 2687-2698.

Gonzaga Vaz Coelho, L. (2000). Latin-American Consensus Conference on Helicobacter pylori infection. The American Journal of Gastroenterology, 95(10), $2688-2691$.

Harris, A., \& Misiewicz, J. J. (2001). ABC of the upper gastrointestinal tract. Management of Helicobacter pylori infection. BMJ (Clinical research ed.), 323(7320), 1047-1050.

Holle, G. E. (1985). 9. Die Pathophysiologie der Ulcuskrankheit. Langenbecks Archiv Für Chirurgie, 366(1), 81-87.

Kappers, J. A. (1960). The development, topographical relations and innervation of the epiphysis cerebri in the albino rat. Zeitschrift For Zellforschung Und Mikroskopische Anatomie, 52(2), 163-215.

Khan, R., Burton, S., Morley, S., Daya, S., \& Potgieter, B. (1990). The effect of melatonin on the formation of gastric stress lesions in rats. Experientia, 46(1), $88-89$.

Klupińska G, Popławski T, Smigielski J, Błasiak J, Chojnacki J. Wpływ melatoniny na oksydacyjne uszkodzenia DNA w komórkach błony śluzowej zoładka u osób z dyspepsja czynnościowa [The effect of melatonin on oxidative DNA damage in gastric mucosa cells of patients with functional dyspepsia]. Pol Merkur Lekarski. 2009 May;26(155):366-9. Polish. PMID: 19606675. 
Konturek, P. C., Konturek, S. J., Brzozowski, T., Dembinski, A., Zembala, M., Mytar, B., \& Hahn, E. G. (1997). Gastroprotective activity of melatonin and its precursor, L-tryptophan, against stress-induced and ischaemia-induced lesions is mediated by scavenge of oxygen radicals. Scandinavian journal of gastroenterology, 32(5), 433-438

Konturek, P. C., Konturek, S. J., Majka, J., Zembala, M., \& Hahn, E. G. (1997). Melatonin affords protection against gastric lesions induced by ischemiareperfusion possibly due to its antioxidant and mucosal microcirculatory effects. European Journal of Pharmacology, 322(1), 73-77.

Konturek P., Brzozwski T., Konturek S. (2011). Gut clock: Implication of circadian rhythms in the gastrointestinal tract. J. Physiol. Pharmacol. (1):139-150.

Konturek PC, Konturek SJ, Celinski K, et al (2010). Role of melatonin in mucosal gastroprotection against aspirin-induced gastric lesions in humans. J Pineal Res, 48, 318-323.

Konturek S.J., Konturek P.C., Brzozowski T., Bubenik G.A. (2007). Role of melatonin in upper gastrointestinal tract. J Physiol Pharmacol, 58 (6), $23-52$.

Konturek, P. C., Celinski, K., Slomka, M., Cichoz-Lach, H., Burnat, G., Naegel, A., Bielanski, W., Konturek, J. W., \& Konturek, S. J. (2008). Melatonin and its precursor L-tryptophan prevent acute gastric mucosal damage induced by aspirin in humans. Journal of physiology and pharmacology: an official journal of the Polish Physiological Society, 59 Suppl 2, 67-75.

Kurata, J. H., \& Abbey, D. E. (1990). The Effect of Chronic Aspirin Use on Duodenal and Gastric Ulcer Hospitalizations. Journal of Clinical Gastroenterology, 12(3), 260-266.

Lastra, C. A., Cabeza, J., Motilva, V., \& Martin, M. J. (1997). Melatonin protects against gastric ischemia-reperfusion injury in rats. Journal of Pineal Research, 23(2), 47-52.

Lin, R., Wang, Z., Cao, J., Gao, T., Dong, Y., \& Chen, Y. (2020). Role of melatonin in intestinal mucosal injury induced by restraint stress in mice. Pharmaceutical biology, 58(1), 342-351.

Ludke, M.; André, M.E.D.A. Pesquisa em educação: abordagem qualitativa. São Paulo: EPU, 1986, p.

Luo, J., Song, J., Zhang, H., Zhang, F., Liu, H., Li, L., Zhang, Z., Chen, L., Zhang, M., Lin, D., Lin, M., \& Zhou, R. (2018). Melatonin mediated Foxp3downregulation decreases cytokines production via the TLR2 and TLR4 pathways in H. pylori infected mice. International immunopharmacology, 64, 116122.

Maganhin, C. C., Carbonel, A. A. F., Hatty, J. H., Fuchs, L. F. P., Oliveira-Júnior, I. S. D., Simões, M. D. J., Simões, R. S., Baracat, E. C., \& Soares-Jr, J. M. (2008). Efeitos da melatonina no sistema genital feminino: breve revisão. Revista Da Associação Médica Brasileira, 54(3), 267-271.

Majumdar, D., Bebb, J., \& Atherton, J. (2011). Helicobacter pylori infection and peptic ulcers. Medicine, 39 (3), 154-161.

Malfertheiner, P., Mégraud, F., O’Morain, C., Hungin, A. P. S., Jones, R., Axon, A., Graham, D. Y., \& Tytgat, G. (2002). Current concepts in the management of Helicobacter pylori infection-The Maastricht 2-2000 Consensus Report. Alimentary Pharmacology \& Therapeutics, 16(2), 167-180.

Mégraud F. et al (1993). Epidemiology of Helicobacter pylori infection. Gastroenterol Clin North Am, 22:73-88

Mertz, H., \& Walsh, J. (1991). Peptic Ulcer Pathophysiology. Medical Clinics of North America, 75(4), 799-814.

Mohamadin, A. M., Ashour, O. M., El-Sherbeny, N. A., Alahdal, A. M., Morsy, G. M., \& Abdel-Naim, A. B. (2009). Melatonin protects against hydrogen peroxide-induced gastric injury in rats. Clinical and experimental pharmacology \& physiology, 36(4), 367-372.

Moraes, S. P. D., Melro, A. P. C., Longo, A., Gun, P. R., Silva, M. A. D. J., \& Reis Neto, J. A. D. (1999). Influência de bloqueadores H2 no processo de cicatrização gástrica. Revista Do Colégio Brasileiro de Cirurgiões, 26(3), 153-155.

Motilva, V., Cabeza, J., \& Alarcón de la Lastra, C. (2001). New issues about melatonin and its effects on the digestive system. Current pharmaceutical design, 7(10), 909-931.

Oderda, G., Vaira, D., Holton, J., Ainley, C., Altare, F., \& Ansaldi, N. (1989). Amoxycillin plus tinidazole for Campylobacter pylori gastritis in children: assessment by serum IgG antibody, pepsinogen I, and gastrin levels. Lancet (London, England), 1(8640), 690-692.

Pihan, G., Majzoubi, D., Haudenschild, C., Trier, J. S., \& Szabo, S. (1986). Early microcirculatory stasis in acute gastric mucosal injury in the rat and prevention by 16,16-dimethyl prostaglandin E2 or sodium thiosulfate. Gastroenterology, 91(6), 1415-1426.

Reiter, R. J., Mayo, J. C., Tan, D. X., Sainz, R. M., Alatorre-Jimenez, M., \& Qin, L. (2016). Melatonin as an antioxidant: under promises but over delivers. Journal of Pineal Research, 61(3), 253-278.

Reiter, R. J.(1991). Pineal Melatonin: Cell Biology of Its Synthesis and of Its Physiological Interactions. Endocrine Reviews, 12(2), 151-180.

Rezende, J., M et al (2009). Úlcera péptica e a ilusão do conhecimento um exemplo de falácia das evidências em medicina. São Paulo: Unifesp.

Rezvanjoo, B., Rashidi, S., Jouyban, A., Beheshtiha, S. H., \& Samini, M. (2010). Effects of vitamin C and melatonin on cysteamine-induced duodenal ulcer in a cholestatic rat model: A controlled experimental study. Current therapeutic research, clinical and experimental, 71(5), 322-330.

Robinwarren, J. (1983). Unidentified Curved Bacilli on Gastric Epithelium in Active Chronic Gastritis. The Lancet, 321 (8336), $1273-1275$.

Samloff I.M., Varis K., Ihamaki T. et al (1982). Relationships among serum pepsinogen 1, sérum pepsinogen II anel gastric mucosal histology, A study in relatives of patients with pernicious anemia. Gastroenterology 83:204-9.

Sener, G., Goren, F. O., Ulusoy, N. B., Ersoy, Y., Arbak, S., \& Dülger, G. A. (2005). Protective effect of melatonin and omeprazole against alendronatinduced gastric damage. Digestive diseases and sciences, 50(8), 1506-1512. 
Sener-Muratoğlu, G., Paskaloğlu, K., Arbak, S., Hürdağ, C., \& Ayanoğlu-Dülger, G. (2001). Protective effect of famotidine, omeprazole, and melatonin against acetylsalicylic acid-induced gastric damage in rats. Digestive diseases and sciences, 46(2), 318-330.

Slomiany, B., Bilski, J., Sarosiek, J., Murty, V., Dworkin, B., VanHorn, K., Zielenski, J., \& Slomiany, A. (1987). Campylobacter pyloridis degrades mucin and undermines gastric mucosal integrity. Biochemical and Biophysical Research Communications, 144(1), 307-314.

Susser, M. (1962). Civilisation and Peptic Ulcer. The Lancet, 279 (7221), 116-119.

Szabo S. (1987). Mechanisms of mucosal injury in the stomach and duodenum: time-sequence analysis of morphologic, functional, biochemical and histochemical studies. Scandinavian journal of gastroenterology. Supplement, 127, 21-28.

Terry, P. D., Villinger, F., Bubenik, G. A., \& Sitaraman, S. V. (2009). Melatonin and ulcerative colitis: Evidence, biological mechanisms, and future research. Inflammatory Bowel Diseases, 15(1), 134-140.

Vollrath L (1981). The Pineal Organ. Heildberg, Germany: Springer-Verlag; p. 659.

Wallace J. L. (1990). Mucosal defense. New avenues for treatment of ulcer disease?. Gastroenterology clinics of North America, 19(1), 87-100.

Younan, F., Pearson, J., Allen, A., \& Venables, C. (1982). Changes in the Structure of the Mucous Gel on the Mucosal Surface of the Stomach in Association with Peptic Ulcer Disease. Gastroenterology, 82(5), 827-831.

Zhang, L., Gong, J. T., Zhang, H. Q., Song, Q. H., Xu, G. H., Cai, L., Tang, X. D., Zhang, H. F., Liu, F. E., Jia, Z. S., \& Zhang, H. W. (2015). Melatonin Attenuates Noise Stress-induced Gastrointestinal Motility Disorder and Gastric Stress Ulcer: Role of Gastrointestinal Hormones and Oxidative Stress in Rats. Journal of neurogastroenterology and motility, 21(2), 189-199. 